\title{
A-Disintegrin and Metalloprotease (ADAM) 10 and 17 Promote Self-renewal of Brain Tumor Sphere Forming Cells.
}

Harry Bulstrode ${ }^{1,2}$, Louise M. Jones ${ }^{1}$, Elodie J. Siney ${ }^{1}$, Jessica M. Sampson ${ }^{1}$, Andreas Ludwig $^{3}$, William P. Gray ${ }^{1,4}$, Sandrine Willaime-Morawek ${ }^{1,5}$

${ }^{1}$ Clinical Neurosciences, Faculty of Medicine, University of Southampton Southampton, SO16 6YD, UK;

${ }^{2}$ Wessex Neurological Centre, Southampton University Hospitals Trust, Southampton, SO16 6YD, UK

${ }^{3}$ Research Group for Vascular Pharmacology, Institute of Pharmacology and Toxicology, RWTH Aachen University, 52074 Aachen, Germany

${ }^{4}$ Present address: Neuroscience and Mental Health Research Institute, Cardiff University, Institute of Psychological Medicine and Clinical Neurosciences, LGF Henry Wellcome Building, Heath Park, Cardiff, CF14 4XN, UK

${ }^{5}$ Corresponding author:

Dr Sandrine Willaime-Morawek

Clinical Neurosciences, Faculty of Medicine, University of Southampton

Room LD72, D Level, South Academic Block, Southampton General Hospital Southampton SO16 6YD, UK

s.willaime-morawek@southampton.ac.uk

Tel: +44 (0)2380 796107; Fax: +44 (0)2380 796085

Author contribution summary:

Harry Bulstrode: conception and design, collection and/or assembly of data, data analysis and interpretation, manuscript writing, final approval of manuscript Louise M. Jones: collection and/or assembly of data, final approval of manuscript Elodie J. Siney: collection and/or assembly of data, final approval of manuscript Jessica M. Sampson: collection and/or assembly of data, final approval of manuscript Andreas Ludwig: provision of study material or patients, final approval of manuscript William P. Gray: conception and design, financial support, provision of study material or patients, data analysis and interpretation, manuscript writing, final approval of manuscript

Sandrine Willaime-Morawek: conception and design, financial support, data analysis and interpretation, manuscript writing, final approval of manuscript

Figures and tables: $\quad 4$

Supplemental materials: $\quad 0$

Pages: 26

Abstract: $\quad 146$ words 


\begin{abstract}
It has been proposed that gliomas contain a subpopulation of 'Brain Tumor Stem Cells' (BTSCs), which demonstrate resistance to conventional therapies. A potential component of the environment governing the behavior of these BTSCs is a class of transmembrane proteins with structural and signaling functions, the A-Disintegrin And Metalloproteases (ADAMs). In this study we confirm overexpression of
\end{abstract}

ADAM10 and 17 in human glioma tissue compared to human controls, and especially in tumor sphere cultures thought to enrich for BTSCs. Inhibition of ADAM10/17 function impairs the growth of tumor spheres with evidence of depletion of the sphere forming cell population. This results from a combination of reduced proliferation, cell death and a switch of sphere-forming cells away from symmetric self-renewal division towards neuronal differentiation. A developing appreciation of the role of ADAMs in BTSC promises insights into pathophysiology and potential therapeutic avenues in this intractable group of tumors.

Keywords: brain tumor, ADAM10, ADAM17

\title{
1. Introduction
}

Gliomas are the most common malignant intrinsic primary brain tumors. The majority are high-grade lesions, with diffuse infiltration that precludes complete surgical resection. Debulking therefore remains the mainstay of management, and despite recent advances in chemotherapy and radiotherapy, median survival from diagnosis remains very short [1]. This limited progress reflects challenges specific to this pathology. 
Firstly, high-grade lesions are genetically heterogeneous across the patient group, with data from The Cancer Genome Atlas suggesting four distinct subtypes [2]. Secondly, the blood brain barrier, where it remains intact, complicates delivery of chemotherapeutics. Finally, the tumor stem cell hypothesis holds that, in gliomas and other tumors, a small subpopulation of the neoplastic cells have tumor initiating capacity based on 'stem' properties of indefinite self-renewal and multipotency [3]. This subgroup of cells exhibits radio- and chemo- resistance, but gives rise to a rapidly-dividing downstream progenitor cell population which contributes to early recurrence after surgery [4]. While prospective identification of Brain Tumour Stem Cells (BTSCs) cells remains a significant challenge, sphere-forming cell populations believed to enrich for the BTSC fraction can be derived from primary brain tumour samples using established techniques.

We have investigated the role of the transmembrane proteins, A-Disintegrin-AndMetalloproteases (ADAMs), a family sharing a structure comprised intracellular protein interaction motifs, and multiple functional extracellular domains. These include an EGF-like sequence, cysteine rich and disintegrin domains, and, of key significance, most incorporate an extracellular metalloprotease active on growth factor ligands and receptors, cytokines and adhesion molecules. Of the many ADAMs identified, ADAM10 and 17 are the most highly conserved, with orthologs demonstrated in yeast, drosophila and primitive chordates [5]. These two ADAMs also have established roles in CNS development, promoting stem cell proliferation and migration [6], and mediating adhesion and neurite outgrowth. These functions, which render ADAM10 and 17 strong candidate regulators of tumor stem cell activity, reflect a range of enzymatic and structural interactions. 
The enzyme domains of ADAM10 and 17 share similar specificity, and activate signaling pro-peptides by cleavage. In the case of ADAM10, this activity applies to Pro-TNF-alpha, Pro-EGF, Notch and Ephrin signaling cascades. These cascades represent key regulatory mechanisms in the control of cell growth and lineage determination, and deregulation of each is a conserved feature across many tumor types $[7 ; 8]$. The protease domain of ADAM17 is also active on a range of growth factors and related receptors, including Pro-TNF-alpha, TNF receptor, Pro-HB-EGF, Notch1, TGF-alpha, IL-6, L-selectin and amyloid precursor protein [5; 9]. The EGF Receptor-PI3 Kinase-Akt pathway appears central to a role for ADAM17 in inducing proliferation and migration [8]. Notch signaling, recognized as essential to maintenance of normal stem cell populations [10], is one example of functional overlap between ADAM10 and ADAM17, with evidence that ADAM10 is essential for ligand induced Notch signaling $[11 ; 12]$, whereas ligand independent signaling is dependent on ADAM17 [13;14]. ADAMs 10 and 17 are also thought to contribute to cell-cell signaling via their disintegrin domain [15], and there is substantial evidence of protease involvement in extracellular matrix degradation associated with tumor invasion[5; 9]' [16]' [17]' [18; 19; 20]

Overexpression of ADAMs 10 and 17, among others, has been reported in gliomas $[21 ; 22]$, as in other cancers. Further, overexpression of ADAM17 correlates with poor prognosis [23] and artificial overexpression of ADAM17 in astrocyte cell lines is sufficient to confer a malignant phenotype including non-adherent growth and increased proliferation and invasion [24], although the mechanism is unknown. Equally, recent evidence points to compensatory upregulation of ADAM expression in breast cancers in response to monoclonal antibody therapies targeted at key ADAM-dependent signaling cascades [25]. 
In this study we have sought to establish the expression profile of ADAM molecules in the sphere-forming cell fraction believed to enrich for BTSCs[26], and their functional significance in the expansion and differentiation of these tumors.

\section{Materials and Methods}

\subsection{Materials}

All procedures followed are in accordance with the ethical standards of the Helsinki declaration $(1964,2008)$ of the World Medical Association. Human tissue was collected according to Ethical Approval obtained from the Berkshire Local Research Ethics Committee (REC 08/H0505/165, CI-WPG), with informed written consent. Tumor samples were obtained at the time of operative resection, selecting material from the enhancing tumor rim by preference from tumors of astrocytic and oligodendroglial lineage, WHO grade II, III and IV. Normal adult temporal cortex tissues were obtained at amygdalohippocampectomy for intractable epilepsy (Southampton and West Hampshire Local research Ethics Committee REC 07/H0504/195, CI-WPG). Embryonic neural tissues (gestation age 7-10 weeks) were obtained at termination of pregnancy (Southampton and West Hampshire Local research Ethics Committee REC 296/00). The selective inhibitor of ADAM10 GI254023X (Inhibition IC50 (ADAM10)/ IC50 (ADAM17) = 102.0), and inhibitor of ADAM10 and 17 GW280264X (Inhibition IC50 (ADAM10)/ IC50 (ADAM17) $=1.4)$ were provided by Prof Andreas Ludwig, Aachen University, Germany [27; 28]. AntiADAM 10 and ADAM 17 antibodies, for use in blocking experiments, were sourced

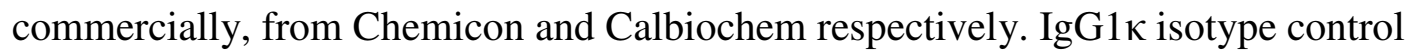
was from Biolegend (\#400101). 


\subsection{Sphere Culture}

On harvesting, all glioblastoma (WHO IV) tissues were enzymatically and mechanically dissociated to single cell suspensions according to established protocols for culture of brain tumor initiating cells [29;30], and cultured in single cell suspension at a density of 10 cells/ $\mu \mathrm{L}$. Growth medium consisted of Neurobasal-A (Invitrogen), supplemented with B27, L-glutamine 2mM, antibiotic/antimycotic preparation, and growth factors (human FGFb10ng/mL, human EGF 20ng/mL and heparin $2 \mu \mathrm{g} / \mathrm{mL})$.

Medium was changed twice weekly and spheres were passaged when the majority exceeded $100 \mu \mathrm{m}$ in diameter. Passaging was accomplished by centrifugation, then 25 cycles of mechanical trituration using a $1 \mathrm{~mL}$ pipette, followed by resuspension at 10 cells $/ \mu \mathrm{L}$ in fresh medium. At the fifth passage, the sphere cell line was considered established, and ready for use in quantitative assays, RNA extraction, or cryopreservation [31]. Sphere counts were performed at day10 unless otherwise specified, and reflect the number of spheres $>100 \mu \mathrm{m}$ in diameter per well, averaged across at least 3 wells per sample.

\subsection{Proliferation and Survival Analysis}

Monolayer cultures were used to assess viability and proliferation 3 days after cell plating. Cells in these cultures were plated at equal live cell density on coverslips coated with $0.0015 \%$ poly-L-ornithine and $50 \mu \mathrm{g} / \mathrm{mL}$ laminin in growth medium. For proliferation, BrdU labeling was used to allow determination of mitotic and labeling indices and was accomplished by exposing the cultures to $20 \mu \mathrm{M}$ BrdU over a period of 2 hours, followed by fixation and immunostaining. Growth fraction and mitotic index were calculated as the proportion of DAPI-labeled cells stained respectively 
with Ki67 and BrdU. Labeling index was calculated as the proportion of Ki67-

labelled cells stained with BrdU. Cell survival and cell death were quantified by using mitotracker green (MTG, $0.1 \mu \mathrm{M}$ ) and propidium iodide (PI, $5 \mu \mathrm{g} / \mathrm{ml})$, respectively. Propidium iodide and nuclear stain 4',6-diamidino-2-phenylindole (DAPI, 20 $\mu \mathrm{g} / \mathrm{ml}$ ) were added to fresh growth media and incubated with live cultures for 40 minutes at $37^{\circ} \mathrm{C}$. This medium was then removed, replaced with MTG-containing medium and incubated at $37^{\circ} \mathrm{C}$ for 40 minutes, before this was replaced with fresh medium for imaging. Cell death was quantified as the proportion of cells DAPI and PI positive, DAPI and MTG positive cells were recorded as live cells, and cells positive for all three markers were depicted as dying cells. Images were averaged across as least three wells per condition.

\subsection{Differentiation Culture}

In order to promote differentiation, spheres were collected by pipetting and triturated to a monocellular suspension. They were then plated on coverslips coated with $0.0015 \%$ poly-L-ornithine and $50 \mu \mathrm{g} / \mathrm{mL}$ laminin, in Neurobasal-A containing $10 \%$ Fetal Bovine Serum. Medium was changed twice weekly and cells were fixed with 4\% paraformaldehyde in PBS after 8 days.

\subsection{RNA extraction and RT-qPCR}

Tissue and sphere samples were stabilized by treatment with RNA Later (Qiagen), and then frozen to $-80^{\circ} \mathrm{C}$ for storage. Extraction of purified RNA was performed using Qiagen's RNeasy Mini kit according to protocol. cDNA was generated using SuperScriptTM III Cells Direct cDNA Synthesis Kit (InvitrogenTM life technologies) and subsequently diluted to $5 \mathrm{ng} / \mu \mathrm{L}$ for $\mathrm{qPCR}$. 
cDNA was amplified using appropriate primers and Precision real-time PCR

MasterMix with SYBRgreen (PrimerDesign Ltd, Southampton) in a thermocycler (Rotor-Gene 6000, Corbett Robotics. Ltd). A panel of 12 housekeeping genes was assessed for suitability using geNorm software (PrimerDesign Ltd, Southampton), and the genes ATP5B and EIF4A2 were selected as the most stable and subsequently used for normalization of all samples tested.

\subsection{Time-lapse Videomicroscopy}

Time-lapse videomicroscopy was performed over 48 hours, at 80 locations (sequences) throughout the wells (40 control, 40 ADAM10/17 inhibition conditions), with visual confirmation of consistent cell density between locations prior to analysis. Monolayer cultures were used with cells plated at equal live cell density on coverslips coated with $0.0015 \%$ poly-L-ornithine and $50 \mu \mathrm{g} / \mathrm{mL}$ laminin in growth medium. The images were analyzed to ascertain the number of divisions per sequence, the number of daughter cells that had migrated from the field of view, and the number of divisions resulting in at least one daughter cell death (which was assessed morphologically by a darkened cell body and lack of movement/contact with other cells). Cell division types (asymmetric or symmetric) and differentiation were analyzed by the use of gridded coverslips to track daughter cells during time-lapse videomicroscopy and subsequent staining with Nestin (stem/progenitor cell marker) and Beta-III-tubulin (early neuronal marker).

\subsection{Immunostaining}

Tissue and sphere $10 \mu \mathrm{m}$ sections were obtained by embedding fragments fixed in 4\%PFA and sectioning on a cryostat. 
Staining was performed on cells and sections using antibodies against Nestin (Millipore, mouse 1:500), GFAP (Dako, rabbit 1:500), Beta-III-tubulin (clone Tuj-1, Sigma, mouse 1:200), ADAM10 (Chemicon, rabbit 1:100), ADAM17 (Calbiochem, rabbit 1:100), BrdU (Serotec, rat 1:200) and Ki-67 (ebioscience.com, mouse 1:500). Secondary antibodies used were Donkey Alexa Fluor 488 and 555 raised against appropriate primary sera (Invitrogen).

For quantification, cell counts were averaged across at least three randomly selected 20x fields.

\subsection{ADAM17 enzymatic activity assay}

The enzyme activity of ADAM17 was quantified by fluorometric analysis using InnoZyme ${ }^{\mathrm{TM}}$ TACE Activity Kit (Calbiochem \#CBA042). To assess inhibitor activity, 10-200ng/ml of purified human TACE protein was incubated on ice for 30 minutes with $2 \mathrm{ug} / \mathrm{ml}$ of anti-ADAM17 (Calbiochem \#PC491) or IgG1к isotype control (Biolegend \#400101) before following the activity kit protocol.

\section{Results}

\subsection{ADAM10 and ADAM17 are highly expressed in high-grade glial tumors, and in} tumor spheres derived from them.

Given that Nestin is highly expressed in dividing stem and progenitor cells, both normal and cancerous, we would expect overexpression in embryonic tissue and in tumors, especially high-grade lesions, compared to normal adult brain tissue. We expect further overexpression in sphere cultures believed to enrich for the stem component. In keeping with this hypothesis, RT-qPCR established Nestin mRNA levels compared to normal cortex samples, at 10.9 (WHO Grade II glioma), 13.9 
(WHO Grade III glioma), 27.8 (WHO Grade IV glioma) and 52.8 (Grade IV glioma spheres) (figure 1A,B), and for controls, 5.6 (embryo tissue) and 25.1 (embryo spheres).

RT-qPCR established ADAM10 mRNA levels compared to normal cortex samples, at 0.8 (WHO Grade II Glioma), 2.1 (WHO Grade III glioma), 2.7 (WHO Grade IV glioma) and 3.2 (Grade IV Glioma spheres; figure 1C,D), and for controls, 1.6 (embryo tissue) and 4.8 (embryo spheres).

ADAM17 mRNA levels were expressed compared to normal cortex samples obtained during resective non tumour epilepsy surgery, at 2.6 (WHO Grade II Glioma), 2.9 (WHO Grade III Glioma), 3.7 (WHO Grade IV Glioma) and 4.2 (Grade IV Glioma spheres; figure 1E,F), and for controls, 0.8 (embryo tissue) and 1.2 (embryo spheres). Immunostaining revealed Nestin staining throughout cultured tumor spheres. Focal high-level expression (figure 1G) with colocalized GFAP staining, a pattern associated with a stem cell component in previous reports, was noted. Similarly CD133 expression was confirmed by staining of tumour and sphere specimens (data not shown). To confirm expression in brain tumor stem/ progenitor cells, immunohistochemistry was performed on WHO Grade IV glioma tissues and tumor spheres for ADAM10, ADAM17 and Nestin (figure 1G,H). Confocal analysis showed colocalization of ADAM10 or 17 with Nestin (higher magnification pictures on figure $1 \mathrm{G}, \mathrm{H})$, both on tissue and spheres, compatible with ADAM10 and 17 expression by BTSCs and/or progenitors. The cellular staining patterns for ADAM10 or 17 are not identical with that of Nestin due to different cellular compartment localization, but coexpression within single cells can be identified. Primary antibody negative controls showed no staining (data not shown). 


\subsection{ADAM10/17 inhibition impairs tumor sphere formation.}

To investigate the role of ADAM10 and 17 in high grade tumor and spheres from glioblastomas, the effects of pharmacological inhibitors of ADAM10/17 function were assessed by counting spheres at culture day 10 . The pharmacological inhibitors used are well-characterized from the standpoint of ADAM-10/-17 inhibition and a few other metalloproteinases [27; 28], Inhibition of ADAM10 alone produced a median reduction in number of spheres across the six cell populations studied to $28 \%$ of baseline, while inhibition of ADAM10/17 together produced a reduction in number of spheres to $14 \%$ of baseline (figure 2A,C). The observed effect was concentration dependent (figure 2A,C), and varied between sphere lineages studied (figure 2B,D). Across six lineages, ADAM17 mRNA levels were a significant predictor of the reduction in sphere numbers in the presence of the ADAM 10/17 inhibitor GW280264X $(10 \mu \mathrm{M})($ Spearman $\mathrm{r}=0.886$, $\mathrm{p}=0.019$ ). The levels of ADAM10 mRNA were not significantly correlated with the decrease in sphere formation induced by ADAM10 inhibition by GI254023X (10 $\mu \mathrm{M})$ (Spearman $\mathrm{r}=0.700, \mathrm{p}=0.188$ ) and by ADAM10/17 inhibition by GW280264X $(10 \mu \mathrm{M})($ Spearman $\mathrm{r}=0.464, \mathrm{p}=0.354)$.

In order to confirm that these results derived from blockade of ADAM10 and 17 function as opposed to non-specific actions of the inhibitors applied, tumor sphere formation was assayed in the presence of blocking antibodies $(2 \mu \mathrm{g} / \mathrm{mL})$ specific to these metalloproteases $[32 ; 33 ; 34 ; 35 ; 36]$, where significant reduction in sphere growth was again observed (figure 2E,F). As control, a non-specific IgG did not have any effect (figure 2G). We also checked that the blocking antibodies indeed decreased ADAM17 enzymatic activity compared to control and non-specific IgG (figure $2 \mathrm{H}$ ). 


\subsection{ADAM10/17 inhibition decreases the survival rate of daughter cells after progenitor cell division.}

A number of mechanisms might account for the observed reduction in sphere formation following ADAM inhibition, including reduced cell survival, reduced proliferation, and/or premature differentiation. Using propidium iodide staining of six primary glioblastoma cultures, we established no difference in overall cell survival in the bulk tumor population at day 3 between control cultures (median 50\%, IQR 2667\%), and those exposed to anti-ADAM10 (median 44\%, IQR 24-78\%) or antiADAM17 (median 53\%, IQR 35-75\%) antibodies.

Next, survival of the dividing cells of the tumor was analyzed using time-lapse videomicroscopy. In the population of daughter cells seen to arise from progenitor divisions, the addition of anti-ADAM10/17 blocking antibodies $(2 \mu \mathrm{g} / \mathrm{mL})$ or the inhibitor GW280264X $(10 \mu \mathrm{M})$ produced a significant increase in cell death over the 48 hour period (figure $3 \mathrm{~A}, \mathrm{~B}$ ).

\subsection{ADAM10/17 inhibition decreases proliferation.}

Growth fraction and mitotic index were calculated as the proportion of DAPI-labeled cells stained with Ki67 and with BrdU respectively, while the labeling index was calculated as the proportion of Ki67-labelled cells stained with BrdU (figure 3C). In ADAM10/17 inhibition conditions, a trend towards reduced division parameters in the bulk population was noted, and when divisions were analyzed with time-lapse videomicroscopy, we confirmed a reduced number of cell divisions over a 48 hour period with ADAM10/17 inhibition (figure 3D). 


\subsection{ADAM10/17 inhibition depletes the stem/progenitor pool by favoring neuronal differentiation over symmetric self-renewal division.}

Under differentiation conditions, a significant increase in the proportion of cells expressing the neuronal lineage marker Beta-III-tubulin, was observed with application of GW280264X (10 $\mu \mathrm{M})$ (figure 4A), pointing toward a more central role of ADAM17 compared to ADAM10 (no effect with GI254023X). There was no significant effect on the overall proportion of cells expressing Nestin or GFAP. Among the Beta-III-tubulin positive cells, around $40 \%$ co-express Nestin (data not shown) which might represent an intermediate state between Nestin positive progenitors and Beta-III-tubulin positive neuroblasts. The increasing trend is seen in both Beta-III-tubulin+Nestin+ and Beta-III-tubulin+Nestin- cell populations (data not shown). These results fit with an effect recently reported in ADAM10 knockout mice [7], which demonstrate premature differentiation of neural stem cells.

To dissect this overall effect, immunostaining of the daughter cells of progenitor divisions (figure 4B,C) identified on time-lapse microscopy was used. This confirmed an increase in the number and proportion of daughter cells expressing Beta-III-tubulin in the presence of ADAM10/17 blocking antibodies $(2 \mu \mathrm{g} / \mathrm{mL})$ compared to control. Importantly, after 2 days in growth condition, the majority of daughter cells continued to express Nestin in control conditions (38 of 40 cells analyzed), but this was not the case when ADAM10/17 were inhibited ( 7 of 24 cells analyzed) (figure 4C). This was confirmed by a decrease in the number of Sox 2 positive cells when ADAM10/17 were inhibited (data not shown). ADAM10/17 inhibition effectively abolished symmetric division of Nestin positive Beta-III-tubulin negative cells (figure 4D) consistent with a fate-determining switch away from maintenance of the Nestin positive BTSC/progenitor cell pool, and consistent with the observed reduction in 
sphere formation. As previously mentioned this effect on the Nestin positive cells was not evident on staining of bulk tumor after 8 days in differentiation conditions (figure 4A), perhaps because Nestin+ BTSCs are outnumbered by a much larger nonproliferating Nestin+ component. An increase in the double negative cell fraction (Nestin- and Beta-III-tubulin -) is also shown: these cells may begin to differentiate without yet expressing Beta-III-tubulin, or express other lineage markers. When analyzing pairs of daughter cells (excluding pairs with death of one of the daughter cells), a switch from symmetric self-renewal division in control conditions to asymmetric differentiating division occurs when ADAM10/17 are inhibited (figure 4D).

Our findings show depletion of the stem/progenitor cell population when ADAM10/17 are inhibited, due to reduced proliferation, a switch towards a asymmetric differentiation division type, daughter cell death, and an increase in neuronal differentiation.

\subsection{ADAM10/17 inhibition impacts on the cell morphology.}

ADAM10 inhibition induced a significant decrease of Beta-III-tubulin+ cells with neurites, and ADAM10/17 inhibition caused a significant reduction in the proportions of Nestin+ cells, GFAP+ cells and Beta-III-tubulin+ cells with processes (figure 4E,F).

\section{Discussion}

The established functions of ADAM10 and ADAM17 are integral to a number of aspects of the neoplastic phenotype, including autonomous growth signaling, invasion and neovascularization $[5 ; 37]$. 
Here we have shown that ADAM10 and ADAM17 are overexpressed in glial tumors, in keeping with expression data from the Rembrandt database [38]. ADAM10 is also overexpressed in embryonic tissue and spheres, but this is expected given the established role in development[7], and indeed is compatible with expression within, and functional significance relating to, the stem cell component, whether normal or cancerous. Immunostaining points to colocalization of ADAM10/17 with the stem and progenitor cell marker Nestin.

We have demonstrated significant impairment of sphere growth associated with inhibition of ADAM10/17 protease function. Further we have shown that this effect is due to changes in survival, proliferation and differentiation. BTSCs stain with Nestin, but are thought to represent a small fraction of the tumor and indeed of the resulting sphere cultures: the majority of Nestin positive cells are likely to represent downstream progenitors [39]. In keeping with a specific impact on the former population, we have shown that overall Nestin staining in the tumor does not decrease with ADAM10/17 inhibition, but that in the daughter cell population identified on time-lapse videomicroscopy, few retain this marker after 48 hours due to a significant reduction in symmetric division of this cell type. We propose that maintenance and expansion of the BTSC pool, which depends on symmetric cell division to produce two Nestin positive Beta-III-tubulin negative daughter cells, is compromised by inhibition of ADAM10 and 17 function (figure 4D). Depletion of the BTSC pool by this mechanism could account for the reduction in divisions observed in these conditions, and indeed impaired sphere growth. We suggest therefore that ADAM10 and/or 17 may be necessary for self-renewal and ultimately maintenance of the brain tumour sphere forming cell population. The involvement of other related enzymes is however not ruled out. 
Despite the staining evidence of premature differentiation, we noted a marked impairment in development of cell processes in the presence of ADAM inhibitors. This agrees with the reported role of ADAM10/17 on neurite growth [40], and points to preservation of this role in the tumor context.

Our study has not addressed the role of ADAMs in the diffuse infiltration and invasion that characterizes high grade gliomas. Both ADAM10 and ADAM17 have also been implicated here, and are likely to exert a combination of effects, on extracellular matrix remodeling, signaling and angiogenesis among others [41; 42]. It is likely that some of the observed variability in ADAM overexpression and effect may be accounted for within emerging molecular classifications of these tumors [43], and investigation of these avenues might be expected to yield insights into pathophysiology, and perhaps ultimately targeted therapeutic approaches. Further work must aim to clarify the role of ADAMs using RNA interference and manipulation of distal components of the signaling pathways involved. Notch signaling, for example, is now a promising therapeutic target [7; 44]. Only through precise characterization of the deregulation of these pathways at all levels can we evaluate the contribution of each ADAM, and establish the potential for pharmacological intervention.

\section{Acknowledgments:}

We are grateful to all the patients who contributed tissue, their families, and to the operating surgeons who obtained these samples, Mr. Paul Grundy and Mr. Jonathan Duffill. We appreciate the contributions of Prof David Wilson, Drs Malik Zaben, Anan Shtaya, and Aminul Ahmed, as well as help from David Johnston for the timelapse experiments. 


\section{Disclosure of potential conflicts of interests:}

None

\section{Financial disclosure:}

MRC Capacity building studentship to E.J.S.; The Nuffield Foundation (URB/38596)

to J.M.S.; EPSRC (DT/E005039/1) and SUHT R\&D (RHM NEU 0133) to W.P.G.;

Wessex Medical Trust (N06) and The Royal Society (RG090173) to S.W.M. The

funders had no role in study design, data collection and analysis, decision to publish or preparation of the manuscript. 


\section{References}

[1]R. Stupp, M.E. Hegi, W.P. Mason, M.J. van den Bent, M.J. Taphoorn, R.C. Janzer, S.K. Ludwin, A. Allgeier, B. Fisher, K. Belanger, P. Hau, A.A. Brandes, J. Gijtenbeek, C. Marosi, C.J. Vecht, K. Mokhtari, P. Wesseling, S. Villa, E. Eisenhauer, T. Gorlia, M. Weller, D. Lacombe, J.G. Cairncross, R.O. Mirimanoff, Effects of radiotherapy with concomitant and adjuvant temozolomide versus radiotherapy alone on survival in glioblastoma in a randomised phase III study: 5-year analysis of the EORTC-NCIC trial. Lancet Oncol 10 (2009) 459-466.

[2]H.S. Phillips, S. Kharbanda, R. Chen, W.F. Forrest, R.H. Soriano, T.D. Wu, A. Misra, J.M. Nigro, H. Colman, L. Soroceanu, P.M. Williams, Z. Modrusan, B.G. Feuerstein, K. Aldape, Molecular subclasses of high-grade glioma predict prognosis, delineate a pattern of disease progression, and resemble stages in neurogenesis. Cancer Cell 9 (2006) 157-173.

[3]H.A. Zaidi, T. Kosztowski, F. DiMeco, A. Quinones-Hinojosa, Origins and clinical implications of the brain tumor stem cell hypothesis. J Neurooncol 93 (2009) 49-60.

[4]T. Hide, T. Takezaki, H. Nakamura, J. Kuratsu, T. Kondo, Brain tumor stem cells as research and treatment targets. Brain Tumor Pathol 25 (2008) 67-72.

[5]D.R. Edwards, M.M. Handsley, C.J. Pennington, The ADAM metalloproteinases. Mol Aspects Med 29 (2008) 258-289.

[6]A. Rubio-Araiz, A. Arevalo-Martin, O. Gomez-Torres, B. Navarro-Galve, D. Garcia-Ovejero, P. Suetterlin, E. Sanchez-Heras, E. Molina-Holgado, F. Molina-Holgado, The endocannabinoid system modulates a transient TNF pathway that induces neural stem cell proliferation. Mol Cell Neurosci 38 (2008) 374-380.

[7]E. Jorissen, J. Prox, C. Bernreuther, S. Weber, R. Schwanbeck, L. Serneels, A. Snellinx, K. Craessaerts, A. Thathiah, I. Tesseur, U. Bartsch, G. Weskamp, C.P. Blobel, M. Glatzel, B. De Strooper, P. Saftig, The disintegrin/metalloproteinase ADAM10 is essential for the establishment of the brain cortex. J Neurosci 30 (2010) 4833-4844.

[8]X. Zheng, F. Jiang, M. Katakowski, Z.G. Zhang, Q.E. Lu, M. Chopp, ADAM17 promotes breast cancer cell malignant phenotype through EGFR-PI3K-AKT activation. Cancer Biol Ther 8 (2009) 1045-1054.

[9]J. Pruessmeyer, A. Ludwig, The good, the bad and the ugly substrates for ADAM10 and ADAM17 in brain pathology, inflammation and cancer. Semin Cell Dev Biol 20 (2009) 164-174.

[10]T.O. Alexson, S. Hitoshi, B.L. Coles, A. Bernstein, D. van der Kooy, Notch signaling is required to maintain all neural stem cell populations--irrespective of spatial or temporal niche. Dev.Neurosci. 28 (2006) 34-48.

[11]G. van Tetering, P. van Diest, I. Verlaan, E. van der Wall, R. Kopan, M. Vooijs, Metalloprotease ADAM10 is required for Notch1 site 2 cleavage. J Biol Chem 284 (2009) 31018-31027.

[12]D. Pan, G.M. Rubin, Kuzbanian controls proteolytic processing of Notch and mediates lateral inhibition during Drosophila and vertebrate neurogenesis. Cell 90 (1997) 271280.

[13]E.C. Bozkulak, G. Weinmaster, Selective use of ADAM10 and ADAM17 in activation of Notch1 signaling. Mol Cell Biol 29 (2009) 5679-5695.

[14]C. Brou, F. Logeat, N. Gupta, C. Bessia, O. LeBail, J.R. Doedens, A. Cumano, P. Roux, R.A. Black, A. Israel, A novel proteolytic cleavage involved in Notch signaling: the role of the disintegrin-metalloprotease TACE. Mol Cell 5 (2000) 207-216.

[15]H. van Goor, W.B. Melenhorst, A.J. Turner, S.T. Holgate, Adamalysins in biology and disease. J Pathol 219 (2009) 277-286.

[16]Z.A. Kohutek, C.G. diPierro, G.T. Redpath, I.M. Hussaini, ADAM-10-mediated N-cadherin cleavage is protein kinase $\mathrm{C}$-alpha dependent and promotes glioblastoma cell migration. J Neurosci 29 (2009) 4605-4615.

[17]G. Weskamp, K. Mendelson, S. Swendeman, S. Le Gall, Y. Ma, S. Lyman, A. Hinoki, S. Eguchi, V. Guaiquil, K. Horiuchi, C.P. Blobel, Pathological neovascularization is reduced by inactivation of ADAM17 in endothelial cells but not in pericytes. Circ Res 106 (2010) 932-940. 
[18]X. Zheng, F. Jiang, M. Katakowski, S.N. Kalkanis, X. Hong, X. Zhang, Z.G. Zhang, H. Yang, M. Chopp, Inhibition of ADAM17 reduces hypoxia-induced brain tumor cell invasiveness. Cancer science 98 (2007) 674-684.

[19]X. Zheng, F. Jiang, M. Katakowski, Y. Lu, M. Chopp, ADAM17 promotes glioma cell malignant phenotype. Mol Carcinog (2011).

[20]Y. Lu, F. Jiang, X. Zheng, M. Katakowski, B. Buller, S.S. To, M. Chopp, TGF-beta1 promotes motility and invasiveness of glioma cells through activation of ADAM17. Oncol Rep 25 (2011) 1329-1335.

[21]T. Kodama, E. Ikeda, A. Okada, T. Ohtsuka, M. Shimoda, T. Shiomi, K. Yoshida, M. Nakada, E. Ohuchi, Y. Okada, ADAM12 is selectively overexpressed in human glioblastomas and is associated with glioblastoma cell proliferation and shedding of heparin-binding epidermal growth factor. Am.J.Pathol. 165 (2004) 1743-1753.

[22]D. Wildeboer, S. Naus, Q.X. my Sang, J.W. Bartsch, A. Pagenstecher, Metalloproteinase disintegrins ADAM8 and ADAM19 are highly regulated in human primary brain tumors and their expression levels and activities are associated with invasiveness. J.Neuropathol.Exp.Neurol. 65 (2006) 516-527.

[23]M.J. Duffy, E. McKiernan, N. O'Donovan, P.M. McGowan, Role of ADAMs in cancer formation and progression. Clin Cancer Res 15 (2009) 1140-1144.

[24]M. Katakowski, F. Jiang, X. Zheng, J.A. Gutierrez, A. Szalad, M. Chopp, Tumorigenicity of cortical astrocyte cell line induced by the protease ADAM17. Cancer Sci 100 (2009) 1597-1604.

[25]M. Gijsen, P. King, T. Perera, P. Parker, B. Larijani, A. Harris, A. Kong, Upregulation of ADAM proteases and HER ligands through a feedback loop mediates acquired resistance to trastuzumab in HER2-amplified breast cancer, in, Breast Cancer Res, 2010, pp. O2.

[26]M. Lenkiewicz, N. Li, S.K. Singh, Culture and Isolation of Brain Tumor Initiating Cells. (2009).

[27]C. Hundhausen, D. Misztela, T.A. Berkhout, N. Broadway, P. Saftig, K. Reiss, D. Hartmann, F. Fahrenholz, R. Postina, V. Matthews, K.J. Kallen, S. Rose-John, A. Ludwig, The disintegrin-like metalloproteinase ADAM10 is involved in constitutive cleavage of CX3CL1 (fractalkine) and regulates CX3CL1-mediated cell-cell adhesion. Blood 102 (2003) 1186-1195.

[28]A. Ludwig, C. Hundhausen, M.H. Lambert, N. Broadway, R.C. Andrews, D.M. Bickett, M.A. Leesnitzer, J.D. Becherer, Metalloproteinase inhibitors for the disintegrin-like metalloproteinases ADAM10 and ADAM17 that differentially block constitutive and phorbol ester-inducible shedding of cell surface molecules. Comb.Chem.High Throughput.Screen. 8 (2005) 161-171.

[29]M. Lenkiewicz, N. Li, S.K. Singh, Culture and isolation of brain tumor initiating cells. Curr Protoc Stem Cell Biol Chapter 3 (2009) Unit3 3.

[30]S.K. Singh, C. Hawkins, I.D. Clarke, J.A. Squire, J. Bayani, T. Hide, R.M. Henkelman, M.D. Cusimano, P.B. Dirks, Identification of human brain tumour initiating cells. Nature 432 (2004) 396-401.

[31]T.M. Fael Al-Mayhani, S.L. Ball, J.W. Zhao, J. Fawcett, K. Ichimura, P.V. Collins, C. Watts, An efficient method for derivation and propagation of glioblastoma cell lines that conserves the molecular profile of their original tumours. J Neurosci Methods 176 (2009) 192-199.

[32]T. Arima, H. Enokida, H. Kubo, I. Kagara, R. Matsuda, K. Toki, H. Nishimura, T. Chiyomaru, S. Tatarano, T. Idesako, K. Nishiyama, M. Nakagawa, Nuclear translocation of ADAM-10 contributes to the pathogenesis and progression of human prostate cancer. Cancer Sci 98 (2007) 1720-1726.

[33]P. Gutwein, A. Schramme, N. Sinke, M.S. Abdel-Bakky, B. Voss, N. Obermuller, K. Doberstein, M. Koziolek, F. Fritzsche, M. Johannsen, K. Jung, H. Schaider, P. Altevogt, A. Ludwig, J. Pfeilschifter, G. Kristiansen, Tumoural CXCL16 expression is a novel prognostic marker of longer survival times in renal cell cancer patients. Eur $\mathrm{J}$ Cancer 45 (2009) 478-489.

[34]R.S. Kenchappa, C. Tep, Z. Korade, S. Urra, F.C. Bronfman, S.O. Yoon, B.D. Carter, p75 neurotrophin receptor-mediated apoptosis in sympathetic neurons involves a biphasic activation of JNK and up-regulation of tumor necrosis factor-alpha-converting enzyme/ADAM17. J Biol Chem 285 (2010) 20358-20368. 
[35]P.H. Kuhn, H. Wang, B. Dislich, A. Colombo, U. Zeitschel, J.W. Ellwart, E. Kremmer, S. Rossner, S.F. Lichtenthaler, ADAM10 is the physiologically relevant, constitutive alpha-secretase of the amyloid precursor protein in primary neurons. EMBO J 29 (2010) 3020-3032.

[36]B.E. Slack, L.K. Ma, C.C. Seah, Constitutive shedding of the amyloid precursor protein ectodomain is up-regulated by tumour necrosis factor-alpha converting enzyme. Biochem J 357 (2001) 787-794.

[37]M. Gooz, ADAM-17: the enzyme that does it all. Crit Rev Biochem Mol Biol 45 (2010) 146169.

[38]N.C. Institute, Rembrandt Database (http://rembrandt.nci.nih.gov), in: N.C. Institute, (Ed.), 2005.

[39]V. Tropepe, M. Sibilia, B.G. Ciruna, J. Rossant, E.F. Wagner, D. van der Kooy, Distinct neural stem cells proliferate in response to EGF and FGF in the developing mouse telencephalon. Dev Biol 208 (1999) 166-188.

[40]T. Maretzky, M. Schulte, A. Ludwig, S. Rose-John, C. Blobel, D. Hartmann, P. Altevogt, P. Saftig, K. Reiss, L1 is sequentially processed by two differently activated metalloproteases and presenilin/gamma-secretase and regulates neural cell adhesion, cell migration, and neurite outgrowth. Mol Cell Biol 25 (2005) 9040-9053.

[41]P. Gooz, M. Gooz, A. Baldys, S. Hoffman, ADAM-17 regulates endothelial cell morphology, proliferation, and in vitro angiogenesis. Biochem Biophys Res Commun 380 (2009) 33-38.

[42]M.L. Moss, A. Stoeck, W. Yan, P.J. Dempsey, ADAM10 as a target for anti-cancer therapy. Curr Pharm Biotechnol 9 (2008) 2-8.

[43]R.G. Verhaak, K.A. Hoadley, E. Purdom, V. Wang, Y. Qi, M.D. Wilkerson, C.R. Miller, L. Ding, T. Golub, J.P. Mesirov, G. Alexe, M. Lawrence, M. O'Kelly, P. Tamayo, B.A. Weir, S. Gabriel, W. Winckler, S. Gupta, L. Jakkula, H.S. Feiler, J.G. Hodgson, C.D. James, J.N. Sarkaria, C. Brennan, A. Kahn, P.T. Spellman, R.K. Wilson, T.P. Speed, J.W. Gray, M. Meyerson, G. Getz, C.M. Perou, D.N. Hayes, Integrated genomic analysis identifies clinically relevant subtypes of glioblastoma characterized by abnormalities in PDGFRA, IDH1, EGFR, and NF1. Cancer Cell 17 (2010) 98-110.

[44]Y. Wu, C. Cain-Hom, L. Choy, T.J. Hagenbeek, G.P. de Leon, Y. Chen, D. Finkle, R. Venook, X. Wu, J. Ridgway, D. Schahin-Reed, G.J. Dow, A. Shelton, S. Stawicki, R.J. Watts, J. Zhang, R. Choy, P. Howard, L. Kadyk, M. Yan, J. Zha, C.A. Callahan, S.G. Hymowitz, C.W. Siebel, Therapeutic antibody targeting of individual Notch receptors. Nature 464 (2010) 1052-1057. 


\section{Figure legends}

Figure 1: ADAM10 and 17 expression in brain tumor tissue and spheres

Relative mRNA levels of Nestin (A and B), ADAM10 (C and D), and ADAM17 (E and F) to adult cortex tissue expression after RT-qPCR. Correlations with the grade of the brain tumors (A, C and E), as well as comparisons between tumor spheres and embryonic spheres (B, D and F) are presented. Data represent $n=3$ (adult tissue), $n=1$ (tissue WHO Grade II), n=3 (tissue WHO Grade III), n=22 (tissue WHO Grade IV), n=11 (spheres WHO Grade IV), $n=9$ (embryo tissue), $n=3$ (embryo spheres) samples. Medians were compared using Kruskal-Wallis followed by Dunn multiple comparison test $* \mathrm{p}<0.05$, and error bars represent interquartile ranges. Images confirm expression of ADAM10 and ADAM17 (green) by immunostaining in tumor spheres (G) and brain tumor tissue WHO Grade IV (H) and confocal colocalization with Nestin (red) and DAPI (blue). Scale bar in upper rows $=25 \mu \mathrm{m}$; in lower rows $=10 \mu \mathrm{m}$. White arrows point to double labeled cells. 


\section{Figure 2: ADAM10/17 inhibition alters tumor sphere formation}

(A) Quantification of the number of spheres per well in the presence of varying concentrations of the ADAM10 inhibitor GI254023X, expressed as percentage of control. Data represent $n=6$ independent samples. Means \pm SEM were compared using Kruskal-Wallis followed by Dunn multiple comparison test. $* \mathrm{p}<0.05$ when compared to control.

(B) Quantification of the number of spheres per well across 6 tumor samples, (002/ to 036/) with or without GI254023X $(10 \mu \mathrm{M})$. Data represent $\mathrm{n}=3$ wells per sample. Means \pm SEM were compared using student T-tests. ${ }^{*} \mathrm{p}=0.008$ for $002 / ; \mathrm{p}=0.383$ for $004 / ;{ }^{*} \mathrm{p}=0.014$ for $005 / ;{ }^{*} \mathrm{p}=0.007$ for $010 / ;{ }^{*} \mathrm{p}=0.002$ for $035 / ; \mathrm{p}=0.01$ for $036 /$ when compared to $0 \mu \mathrm{M}$.

(C) Quantification of the number of spheres per well in the presence of varying concentrations of the ADAM10/17 inhibitor (GW280264X), expressed as percentage of control. Data represent $n=6$ independent samples. Means \pm SEM were compared using Kruskal-Wallis followed by Dunn multiple comparison test. ${ }^{*} \mathrm{p}<0.05$ when compared to control or $0.1 \mu \mathrm{M}$.

(D) Quantification of the number of spheres per well across 6 tumor samples, (002/ to $036 /)$ with or without GW280264X $(10 \mu \mathrm{M})$. Data represent $\mathrm{n}=3$ wells per sample. Means \pm SEM were compared using student T-tests. ${ }^{*} \mathrm{p}=0.036$ for $002 / ; \mathrm{p}=0.03$ for $004 / ; \mathrm{p}=0.127$ for $005 / ;{ }^{*} \mathrm{p}=0.003$ for $010 / ; \mathrm{p}=0.1$ for $035 /$ (Mann Withney test); ${ }^{*} \mathrm{p}=0.006$ for $036 /$ when compared to $0 \mu \mathrm{M}$.

(E) Quantification of the number of spheres per well for sample 004/ with or without anti-ADAM10 or ADAM17 antibodies $(2 \mu \mathrm{g} / \mathrm{mL})$. Data represent $\mathrm{n}=6$ wells per group. Means \pm SEM were compared using one way ANOVA 
followed by Tukey multiple comparison test $* \mathrm{p}=0.001$ when compared to control.

(F) Quantification of the number of spheres per well for sample 005/ with or without anti-ADAM10 or ADAM17 antibodies $(2 \mu \mathrm{g} / \mathrm{mL})$. Data represent $\mathrm{n}=6$ wells per group. Means \pm SEM were compared using one way ANOVA followed by Tukey multiple comparison test $* \mathrm{p}=0.039$ when compared to control.

(G) Quantification of the number of spheres per well for sample $037 /$ with or without non-specific IgG, or anti-ADAM10 and 17 antibodies $(2 \mu \mathrm{g} / \mathrm{mL})$. Data represent $n=3-6$ wells per group. Means \pm SEM were compared using one way ANOVA followed by Bonferonni multiple comparison test $* \mathrm{p}<0.001$ when compared to control or IgG.

(H) Quantification of the ADAM17 enzymatic activity with or without nonspecific IgG, or anti-ADAM17 antibody $(2 \mu \mathrm{g} / \mathrm{mL})$. Data represent $\mathrm{n}=3-5$ wells per group. Means \pm SEM were compared using student t-test $* \mathrm{p}=0.003$ for left graph and $* \mathrm{p}=0.039$ for right graph when compared to control or IgG respectively. 
Figure 3: ADAM10/17 inhibition decreases the survival rate of daughter cells and the proliferation rate of progenitors

(A) Sample time-lapse videomicroscopy images demonstrating a progenitor cell division with the 2 corresponding daughter cells (arrows, left and middle) followed by death of a daughter cell (right, horizontal arrow).

(B) Daughter cell death rates following progenitor cell division over a 48 hour time-lapse videomicroscopy experiment with ADAM 10 and 17 blocking antibodies ( $2 \mu \mathrm{g} / \mathrm{mL})$ (61 fields) and control (57 fields) conditions are compared. Cell death in the daughter cell population is significantly increased by ADAM10/17 inhibition. $\left(\chi^{2}=8.343, \mathrm{df}=1, * \mathrm{p}=0.004\right)$

(C) The mitotic index measures the proportion of cells (DAPI+) which are in S phase $(\mathrm{BrdU}+)$ at day 3 in culture (one way ANOVA $\mathrm{p}=0.265$ ). The growth fraction indicates the proportion of cells (DAPI+) which are proliferating (Ki67+) (one way ANOVA p=0.797). The labeling index quantifies the proportion of proliferating cells (Ki67+) which are in S-phase (BrdU+) (one way ANOVA $\mathrm{p}=0.310$ ). Data represent $n=4$ independent samples.

(D) The total number of divisions observed over a 48 hour period with ADAM 10 and 17 blocking antibodies $(2 \mu \mathrm{g} / \mathrm{mL})$ and control conditions are compared. Uniform plating density between conditions was confirmed on the initial images obtained. Significantly fewer divisions occur in ADAM10/17 inhibition conditions (Mann Whitney $\mathrm{U}=1373.500$, * $\mathrm{p}=0.047$ ). 
Figure 4: ADAM10/17 inhibition directs brain tumor stem cells away from

\section{symmetrical expansion and towards neuronal differentiation}

(A) Quantification of Nestin, GFAP and Beta-III-tubulin positive cells after 8 days in differentiation culture conditions, as a percentage of the total number of cells quantified using DAPI. Data represent quantification of 3 fields/sample, $\mathrm{n}=3$ samples. Mean \pm SEM were compared using one way ANOVA followed by Holm-Sidak multiple comparison test. ${ }^{*} \mathrm{p}<0.001$ when compared to control or GI254023X.

(B) Representative images obtained using time-lapse videomicroscopy illustrate a cell division, with the progeny (arrows) subsequently fixed and co-stained for Beta-III-tubulin (green) and Nestin (red).

(C) The Beta-III-tubulin (Beta-III-T) and Nestin co-staining of the daughter cells generated during 48 hours of time-lapse imaging was examined in control conditions $(\mathrm{n}=40)$, and in the presence of ADAM 10 and 17 blocking antibodies $(2 \mu \mathrm{g} / \mathrm{mL})(\mathrm{n}=24)$. In inhibition conditions, a substantial decrease in the Beta-III-tubulin- Nestin+ stem and progenitor daughter cell fraction is evident, and the proportion of Beta-III-tubulin + cells is increased $\left(\chi^{2}=38.398\right.$, $\mathrm{df}=3, \mathrm{p}<0.001)$.

(D) Surviving pairs of daughter cells (excluding pairs with death of either daughter cell) from time-lapse videomicroscopy were analyzed in control conditions ( $\mathrm{n}=15$ pairs) and ADAM10/17 inhibition ( $\mathrm{n}=9$ pairs). Inhibition conditions are associated with abolition of symmetrical cell division by the Nestin+ Beta-III-tubulin- component corresponding to BTSCs (Fisher exact test $=20.181, \mathrm{p}<0.001)$. 
(E) Representative images illustrate the staining results quantified in (A) and (F): Beta-III-tubulin (green), GFAP (red), Nestin (green), DAPI (blue) are stained in control conditions or with GW280264X $(10 \mu \mathrm{M})$. Scale bar $=25 \mu \mathrm{m}$.

(F) Quantification of cells with a differentiated morphology (presence of long processes) after 8 days in differentiation culture conditions, as percent of numbers of cells positive for Nestin, GFAP or Beta-III-tubulin. Data represent quantification of 3 fields/sample, $n=3$ samples. Mean \pm SEM were compared using Kruskal-Wallis (for Nestin and GFAP) or one way ANOVA (for BetaIII-tubulin) followed by Tukey multiple comparison test. ${ }^{*} \mathrm{p}<0.05$ when compared to control; **p $<0.05$ when compared to control or GI254023X. 
Figure 1

Click here to download high resolution image
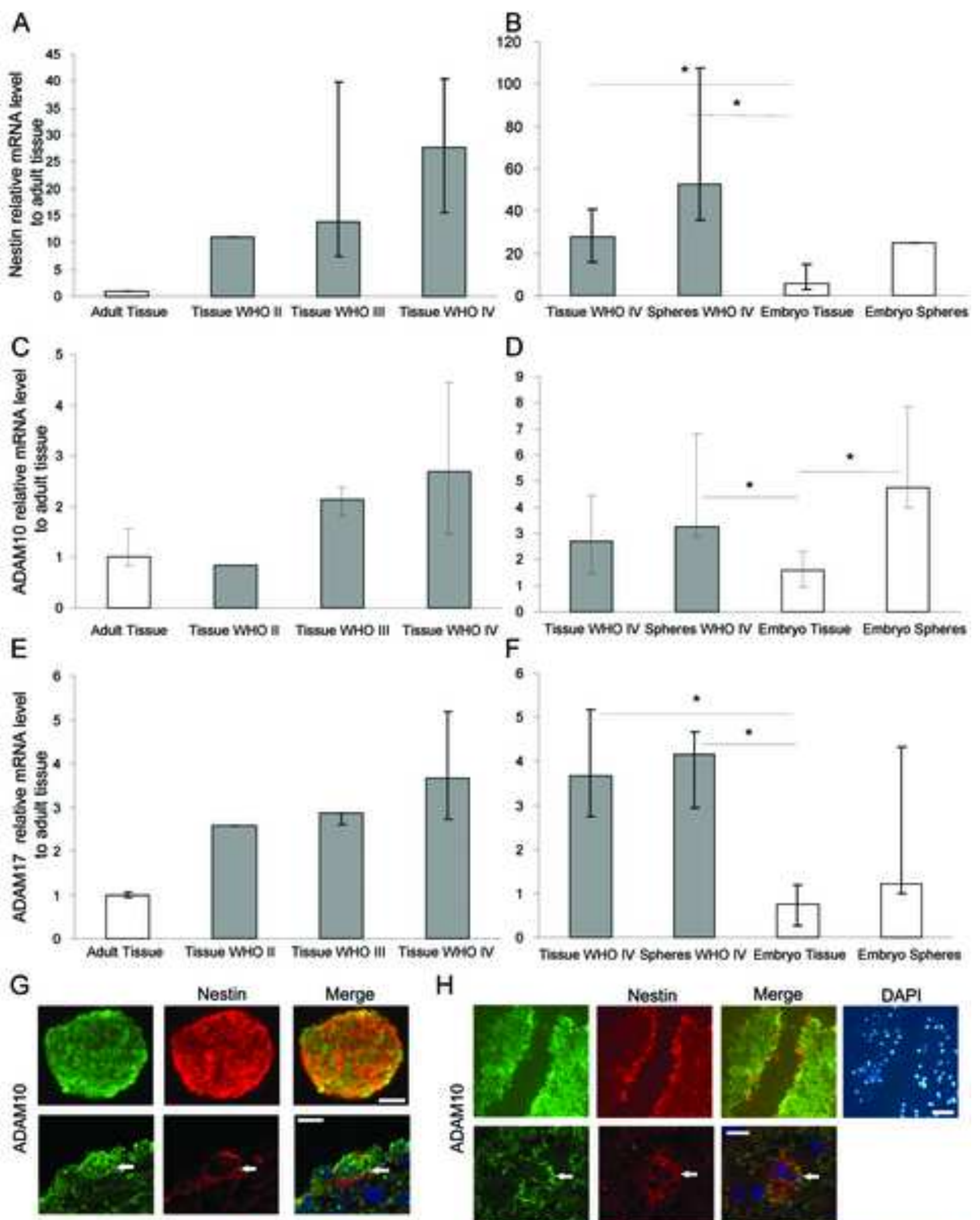

$\mathrm{H}$
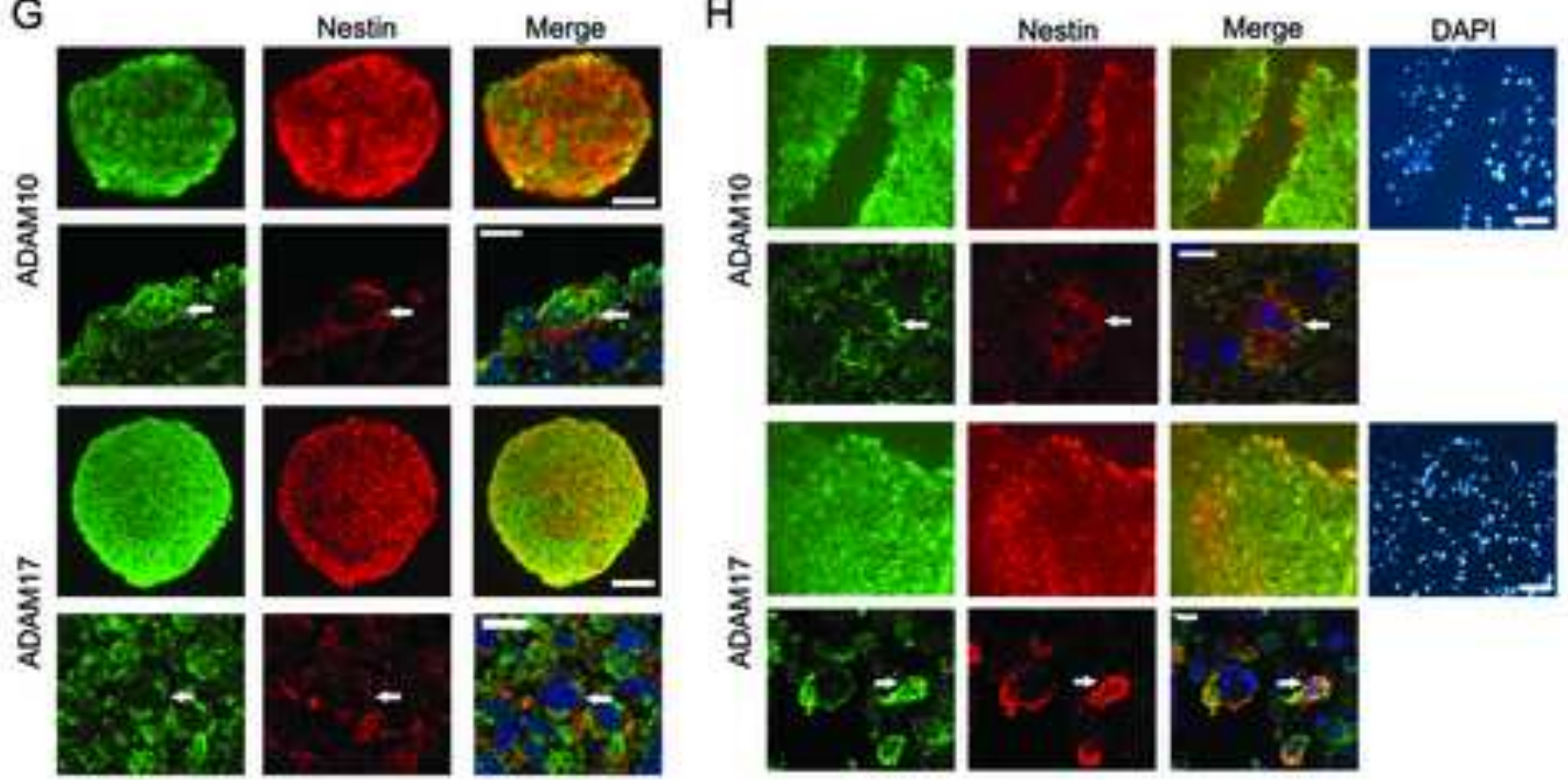
Figure 2

Click here to download high resolution image

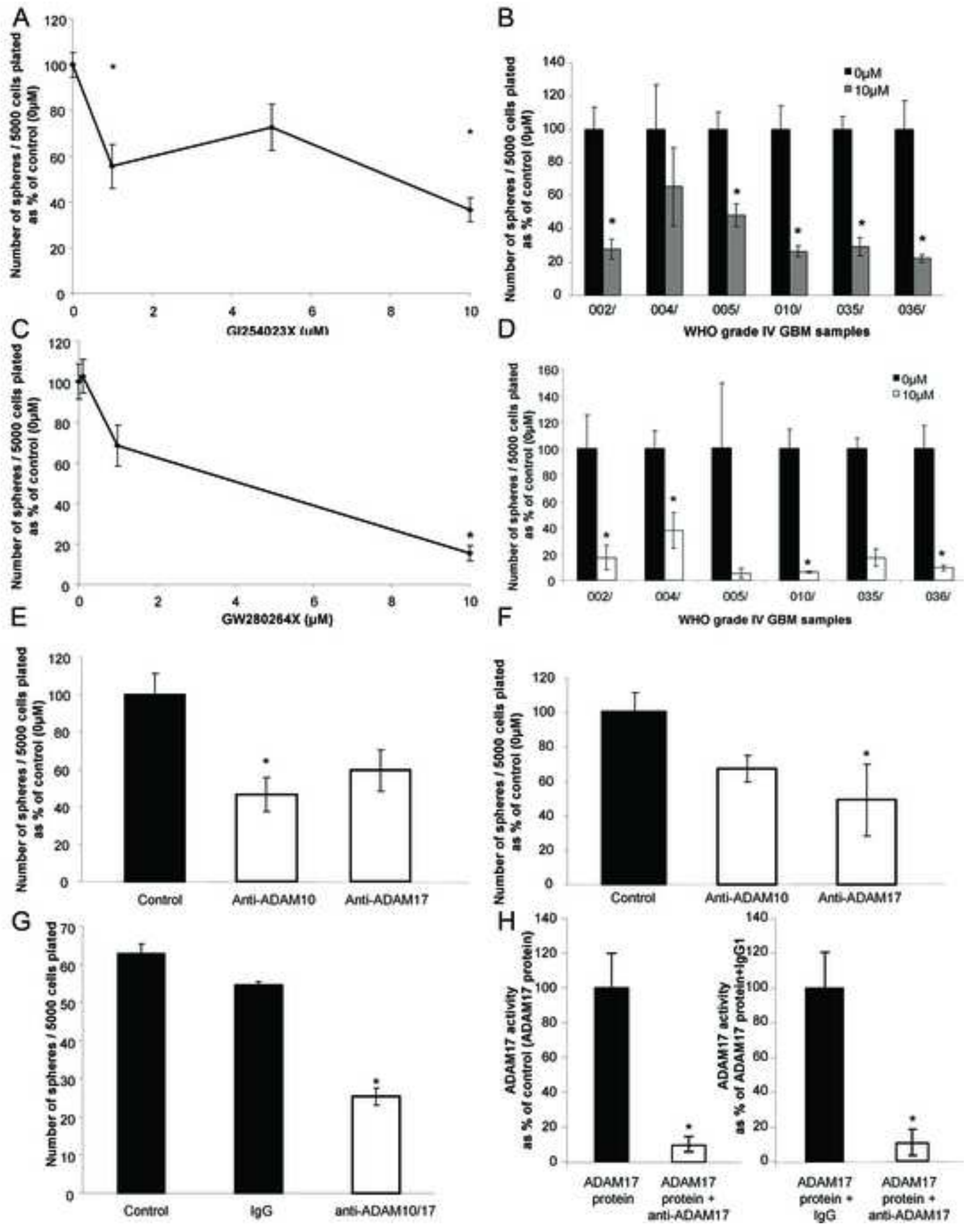


Click here to download high resolution image

A

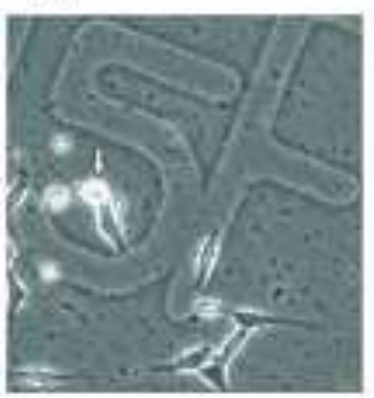

C 0.

$\begin{array}{ll} & 0 . \\ & 0 . \\ & 0.3\end{array}$

0.2

0.1

0

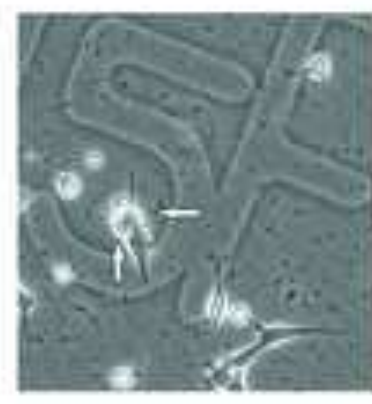

- Control

- Gi24 $4023 \mathrm{X}$

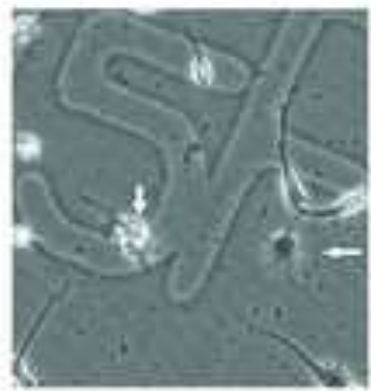

B

\begin{tabular}{|c|c|c|c|}
\hline & $\begin{array}{c}\text { Number of } \\
\text { divisions with } \\
\text { desth of a } \\
\text { daughter cell }\end{array}$ & $\begin{array}{c}\text { Total number } \\
\text { of divisions } \\
\text { analyzed }\end{array}$ & $\begin{array}{c}\% \text { of divisions } \\
\text { with death of a } \\
\text { daughter cell }\end{array}$ \\
\hline Control & 2 & 106 & $1,89 \%$ \\
\hline $\begin{array}{c}\text { ADAM10/17 } \\
\text { Inhibition }\end{array}$ & 10 & 70 & $14,3 \%$ \\
\hline
\end{tabular}

D

\begin{tabular}{|c|c|c|c|}
\hline & $\begin{array}{c}\text { Number of } \\
\text { dlvisions }\end{array}$ & $\begin{array}{c}\text { Number of } \\
\text { fields } \\
\text { analyzed }\end{array}$ & $\begin{array}{c}\text { Median, IQR } \\
\text { of divisions } \\
\text { per fleid }\end{array}$ \\
\hline Control & 177 & 57 & $2,1-5$ \\
\hline $\begin{array}{c}\text { ADAM1017 } \\
\text { inhibition* }\end{array}$ & 132 & 61 & $2,1-3$ \\
\hline
\end{tabular}


Figure
Click here to download high resolution image
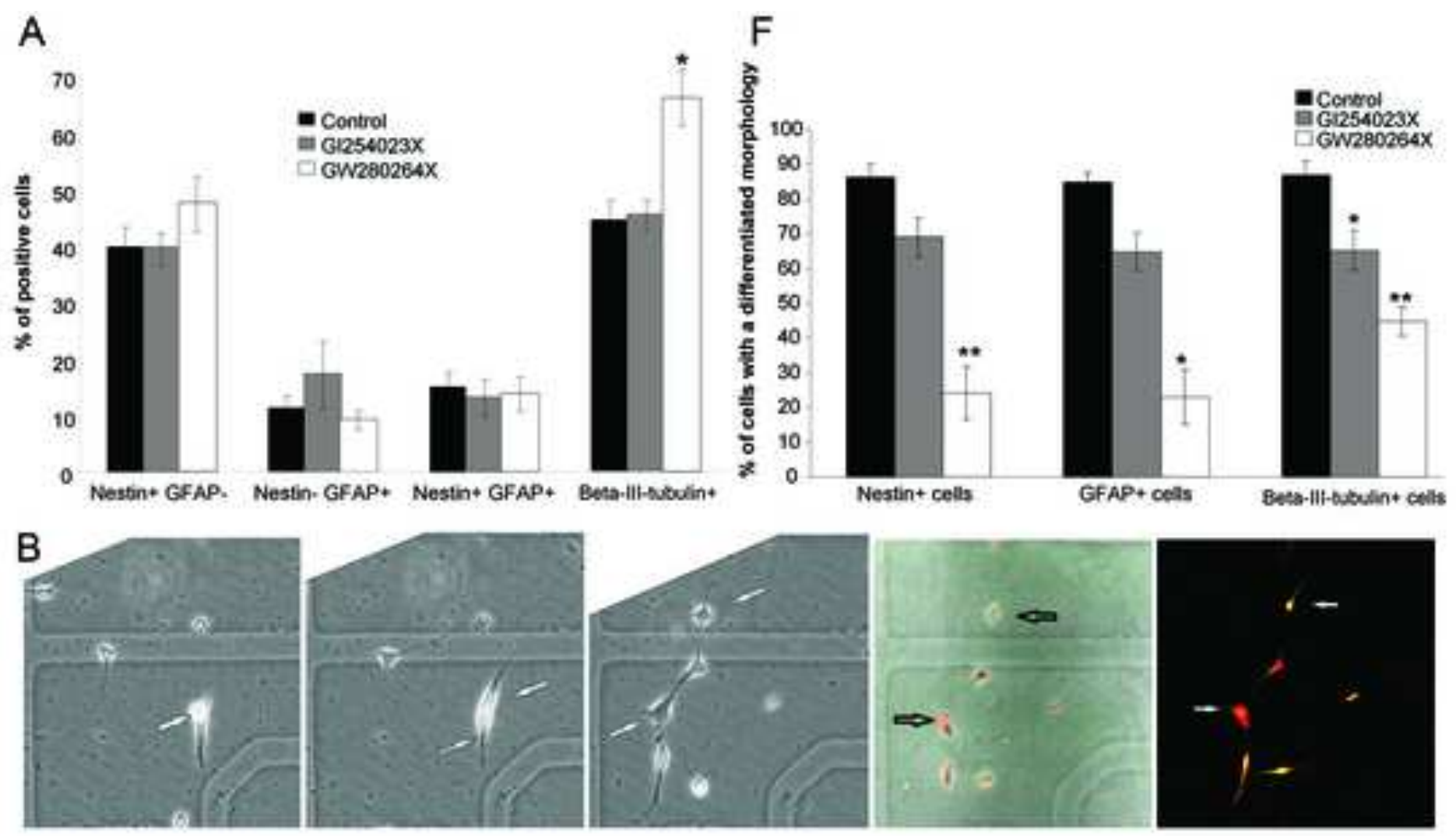

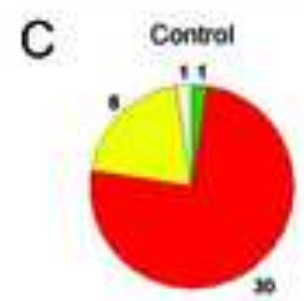

$n=40$

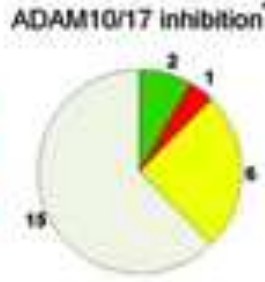

$n=24$

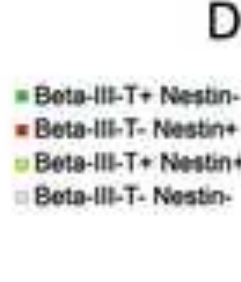

GFAP
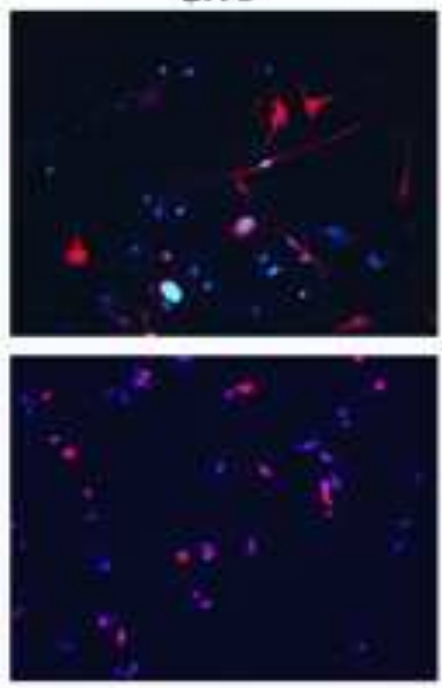

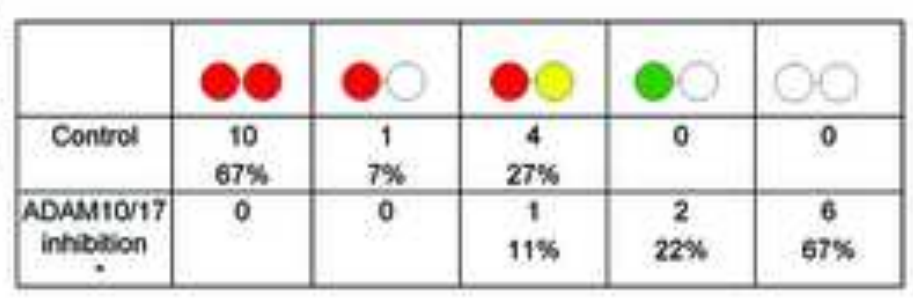

Nestin
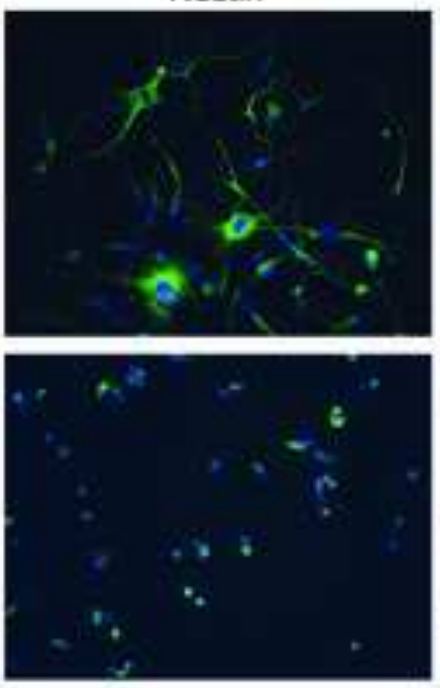
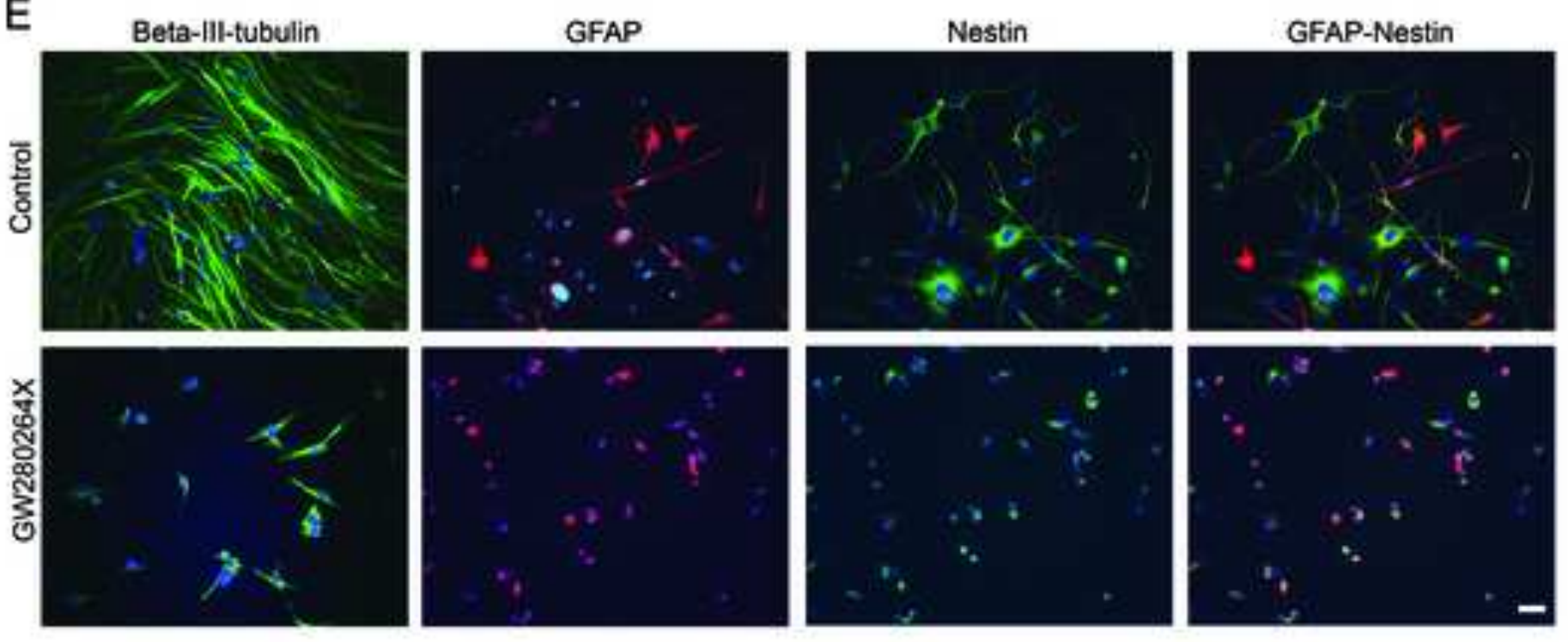